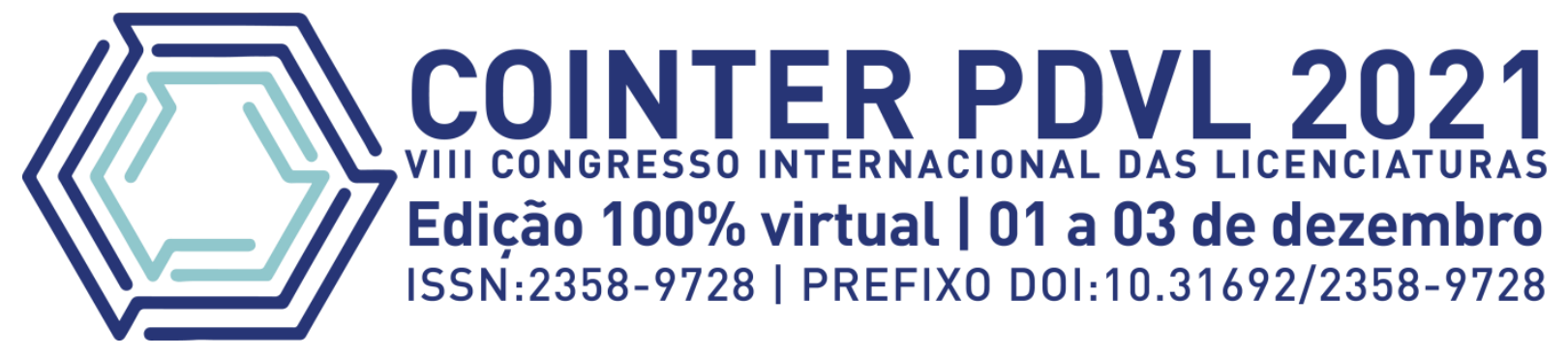

QUIMI-CANTO: TRABALHO INTERDISCIPLINAR DE APRENDIZAGEM BASEADO EM PROJETOS DE DIVULGAÇÃO CIENTÍFICA NA TEMÁTICA DE USO DE COMBUSTÍVEL FÓSSIL

\title{
QUIMI-CANTO: TRABAJO INTERDISCIPLINAR DE APRENDIZAJE BASADO EN PROYECTOS DE DIVULGACIÓN CIENTÍFICA EN LA TEMÁTICA DE USO DE COMBUSTIBLE FÓSIL
}

\section{QUIMI-CANTO: INTERDISCIPLINARY WORK TO PROJECT-BASED LEARNING TO SCIENCE DIVULGATION ON THE THEME USE OF FOSSIL FUEL}

\author{
Apresentação: Relato de Experiência \\ Mg (c) Ignacio Fuentes ${ }^{1}$; Dra. Lastenia Ugalde ${ }^{2}$
}

\section{INTRODUCCIÓN}

Los cambios en educación suelen ser graduales y lentos en comparación a la producción de otros ámbitos de la vida cotidiana. Cada generación es distinta una de otras desde su forma de afrontar los desafíos cotidianos, así como los desafíos basados en necesidades globales, lo que ha obligado a los organismos educativos a estar atentos frente a constantes modificaciones. Las metodologías más ligadas al constructivismo han contribuido con un papel protagónico en sus modificaciones, llevando al Estado de Chile a realizar un proceso de modificación progresivo, cuya implementación en su totalidad se terminó de implementar el año 2020.

Esta modificación ha implicado, una disminución de las horas de ciencias en plan común, pasando de 4 a 2 horas semanales, y transformando su nombre a Ciencias para la ciudadanía con una dirección a una integración de las asignaturas de Física, Química y Biología. También se destaca el aumento de la variedad de asignaturas electivas de profundización a las cuales puede optar cada estudiante, incluyendo en el área de ciencias, asignaturas principalmente direccionadas al área de la biología como Ciencias de la salud, Biología de ecosistemas, Biología Celular y molecular, y con la aparición simple de Química y Física (UCE, 2019). Si se analiza la escolarización chilena en general, se observa una visión fragmentada y

\footnotetext{
${ }^{1}$ Magister (c) en Enseñanza de las Ciencias, Universidad de Playa Ancha, ignaciofuentes@alumnos.upla.cl

2 Académica asociada Universidad de Playa Ancha, lastenia.ugalde@upla.cl
} 


\section{QUIMI-CANTO: TRABALHO INTERDISCIPLINAR DE APRENDIZAGEM}

sectorizada de la educación, la cual ha estado arraigada en el sistema educacional hace bastante tiempo (Catañer, 1998).

La presentación de estos nuevos programas curriculares ha permitido una inclusión tanto de los nuevos marcos legales establecidos progresivamente desde la Ley General de Educación (Ley 20370, 2009) hasta la proposición de metodologías ligadas al constructivismo, como es el Aprendizaje Basado a Proyectos, cuya implementación propiciaría el desarrollo de Contenidos, Habilidades y Actitudes.

El aprendizaje basado en proyectos (ABP), involucra no solo la realización de un producto final, sino que también el desarrollo de diferentes competencias transversales tales como el trabajo colaborativo, la discusión y toma de decisiones, entre otras (Vilá, 2014) las cuales involucra en su particularidad, la realización de un proyecto para la resolución de un problema, aplicando habilidades y conocimientos adquiridos, la disposición de mayor autonomía y responsabilidad ante la propia formación del estudiante (De Miguel, 2006). Del mismo modo, se potencia la idea de aplicar esta metodología con una mirada centrada en el desarrollo del aprendizaje por sobre el desarrollo de la enseñanza (Tünnerman, 2011), enfrentándolos a situaciones de contextos que le competen y que lo involucren en torno a un trabajo colaborativo acompañado de una investigación asociada a una planificación, diseño y desarrollo de un producto final, utilizando los aprendizajes adquiridos (Girón,2014). Como característica final, el ABP debe tener una visión interdisciplinaria utilizando elementos tecnológicos, para que, de esta manera, se puedan establecer mayores grados de motivación y propiciar la construcción de aprendizajes significativos. (Glasserman, 2010).

\section{INFORME DE EXPERIENCIA}

Bajo una visión aterrizada de la diversa realidad escolar chilena, no se había propiciado el desarrollo de esta metodología hasta este último cambio curricular antes mencionado, incluyendo principalmente la educación en ciencias, donde la preponderancia de las metodologías tradicionales, el bajo desarrollo de la indagación científica escolar e incluso la necesidad de manejar de mejor manera los elementos asociados a la educación como la evaluación, la planificación y la implementación de metodologías más constructivistas en el aula, se han transformado en verdaderos obstáculos para la gestión docente (Cofré, 2010), sin mencionar la falta de horas o la baja cantidad de espacios colaborativos con otros docentes para un trabajo interdisciplinario. En razón de esta problemática se plantea El diseño de la investigación basado en la unidad didáctica "Quími-Canto", que tiene por finalidad el realizar un proyecto de divulgación científica con estudiantes de segundo año de enseñanza media 
científico-humanista, teniendo como base una investigación en torno a la unidad de química orgánica, específicamente la temática de uso de combustible fósil, se espera como producto final del proyecto, un material audiovisual en formato de videoclip, de una canción que verá modificada su letra con los conceptos de la temática indagada por los estudiantes.

Esta propuesta será trabajada con tres docentes de un establecimiento particular subvencionado científico-humanista de la comuna de Macul, Región Metropolitana, quienes acogerán la propuesta y realizarán las modificaciones correspondientes en conjunto para su posterior implementación, de tal modo que se puedan propiciar los espacios colaborativos interdisciplinarios y enriquecer la propuesta inicial y a su vez, permitir analizar los factores involucrados al momento de diseñar dichas actividades, con el objetivo de resolver la pregunta de investigación ¿Cuáles son los factores que generan mayor dificultad entre los docentes en diseño una actividad interdisciplinaria, con una metodología de aprendizaje orientado a proyectos?

Para esta investigación que está planificada para ejecutarse durante el tercer trimestre del presente año, se utilizará una metodología no experimental, de carácter cualitativo correlacional e interpretativo, establecido en base a intervenciones con una muestra de 3 docentes (1 por cada área involucrada) del establecimiento educacional intervenido, involucrando las etapas principales de la investigación que se observan en la figura 1.

Figura 1. Etapas de implementación del proyecto

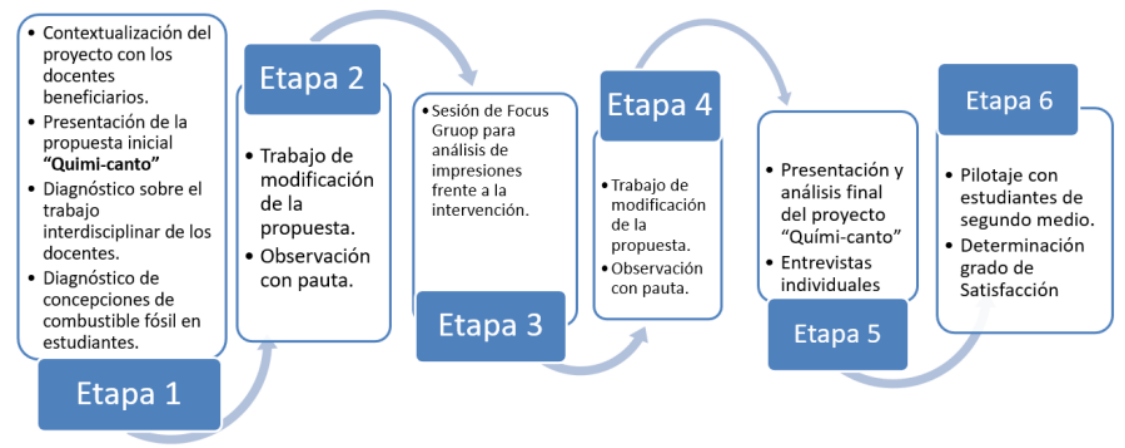

Fuente: Propia

Para este proyecto se espera realizar un tratamiento de datos de fuente primaria en base a las entrevistas a los docentes, así como de la correlación entre las declaraciones con su accionar al trabajar interdisciplinariamente. Finalmente se medirá el grado de satisfacción de un grupo piloto de estudiantes que implementarán esta metodología. 


\section{CONCLUSIONES}

La propuesta presenta una potencialidad de amplitud en torno a la propuesta Quimicanto, expandiéndola a otros niveles o unidades de la asignatura de química y que se pueda mantener la vinculación con las áreas de Artes y Música, así como también impulsar el desarrollo de la creatividad y proponer la inclusión de otros docentes a trabajos colaborativos interdisciplinarios en el sistema escolar tradicional con la metodología de Aprendizaje Basado en Proyectos. Al mismo tiempo, la implementación de este tipo de actividades podrá colaborar con la motivación de los estudiantes en continuar sus estudios ligados a la ciencia y en específico a la química. Finalmente hay que destacar este proyecto como una oportunidad de lograr un aprendizaje significativo en periodo de pandemia y educación remota.

\section{REFERENCIAS}

CASTAÑER, M; TRIGO,E. La interdisciplinariedad en la Enseñanza Secundaria Obligatoria. Inde Publicaciones. Zaragoza, España. 1998

COFRÉ, H ET.AL. La educación científica en Chile: Debilidades de la enseñanza y futuros desafíos de la educación de profesores de ciencia. Estudios Pedagógicos. V.36 n.2 Valdivia, Chile. 2010

DE MIGUEL. M. (Coord.). Metodologías de enseñanza y aprendizaje para el desarrollo de competencias. Orientaciones para el profesorado universitario ante el espacio europeo de educación superior. Madrid: Alianza Editorial. 2006

GIRÓN, M. Puesta en marcha del método enseñanza - aprendizaje "Aprendizaje Orientado a Proyectos (AOP)". VII Coloquio Internacional sobre la Enseñanza de las Matemáticas" Universidad Peruana de las Ciencias aplicadas. Lima. 2014

GLASSERMAN, L.; RESÉNDIZ, M.; RIQUELME, J. Aprendizaje Orientado a Proyectos como apoyo para la integración de asignaturas en la formación profesional. Apertura. Vol. 2, núm. 2. Universidad de Guadalajara, México. 2010

TÜNNERMANN, C. El constructivismo y el aprendizaje de los estudiantes. Universidades, UDAL, México. pp 21 -32. 2011

UNIDAD DE CURRICULUM Y EVALUACIÓN. Bases Curriculares $3^{\circ}$ y $4^{\circ}$ Medio. Ministerio de Educación. Chile.2020

VILÁ, R.; RUBIO, M.; BERLANGA, V. La investigación formativa a través del aprendizaje orientado a proyectos: Una propuesta de innovación en el grado de pedagogía. Innovación educativa, ${ }^{\circ} 23$, pp 241-258. 2014 